\title{
Myogenin is a Specific Marker for Rhabdomyosarcoma: An Immunohistochemical Study in Paraffin-Embedded Tissues
}

S. Kumar, M.D., E. Perlman, M.D., C.A. Harris, B.Sc., M. Raffeld, M.D., M. Tsokos, M.D.

Laboratory of Pathology, National Cancer Institute, National Institutes of Health, Bethesda Maryland (SK, CAH, MR, MT), and Johns Hopkins Hospital, Baltimore, Maryland (EP)

Myogenin belongs to a group of myogenic regulatory proteins whose expression determines commitment and differentiation of primitive mesenchymal cells into skeletal muscle. The expression of myogenin has been demonstrated to be extremely specific for rhabdomyoblastic differentiation, which makes it a useful marker in the differential diagnosis of rhabdomyosarcomas (RMS) from other malignant small round cell tumors of childhood. Commercially available antibodies capable of detecting myogenin in routinely processed formalin-fixed paraffin-embedded (FFPE) tissue are now available. In this study, we evaluated myogenin expression using the monoclonal myf-4 antibody (Novocastra Labs) on FFPE in a large number of pediatric tumors in order to define the clinical utility of this marker. A total of 119 tumors were studied. These included 48 alveolar RMS (ARMS), 20 embryonal RMS (ERMS), one spindle cell RMS, 16 Ewing's sarcomas (ES), six nephroblastomas, two ectomesenchymomas, seven precursor hematopoietic neoplasms, five olfactory neuroblastomas, three neuroblastomas, six desmoplastic small round cell tumors, and five rhabdoid tumors. Distinct nuclear staining for myogenin was noted in all 69 RMS. Notably, the number of positive tumor cells differed between the ARMS and ERMS. In ARMS, the majority of tumor cells (75 to $100 \%$ ) were positive, in contrast to ERMS, in which the positivity ranged from rare + to $25 \%$ in all but three tumors. Additionally, myogenin positivity was seen in two of two ectomesenchymomas and in two nephroblastomas with myogenous differentiation. All other tumors were clearly negative. Our results indicate that staining for myogenin is an extremely reliable and

Copyright $(\odot 2000$ by The United States and Canadian Academy of Pathology, Inc.

VOL. 13, NO. 9, P. 988, 2000 Printed in the U.S.A.

Date of acceptance: April 6, 2000.

Address reprint requests to: Shimareet Kumar, M.D., Anatomic Pathology,

Children's National Medical Center, 111 Michigan Avenue, NW, Washing-

ton, DC 20010-2970; fax: 202-884-4030. specific marker for rhabdomyoblastic differentiation. It gives consistent and easily interpretable results in routinely fixed tissues.

KEY WORDS: Myogenin, Rhabdomyosarcoma, Paraffin-embedded tissue, Immunohistochemistry. Mod Pathol 2000;13(9):988-993

The differential diagnosis of rhabdomyosarcomas (RMS) from other poorly differentiated pediatric round cell tumors, including the Ewing's sarcoma family of tumors (ESFT), neuroblastomas, and hematopoietic neoplasms can be difficult on histologic grounds alone, although the use of an antibody panel including mic-2, desmin, and hematopoietic lineagespecific antibodies now allows an accurate diagnosis in the majority of cases. The expression of markers specific for rhabdomyoblastic differentiation is particularly useful in defining lineage in some poorly differentiated/undifferentiated tumors and antibodies to MyoD1 and myogenin proteins have been found to be of most use in this regard (1-5). Although staining for MyoD1 was the first to be used in the diagnostic setting, nonspecific cytoplasmic staining often makes interpretation of results problematic when fixed tissue sections are used $(3,6,7)$. Antibodies to myf- 4 , the human homologue of myogenin, are also available and have been evaluated in a few studies $(3,6-8)$. However, these studies have included relatively few cases of RMS, particularly of alveolar subtype $(3,6,8)$ or are reported only in abstract form (6). In addition, some have been performed only on frozen sections (9). In this study, we report our findings with a new, commercially available anti-myogenin antibody in a large series of RMS and other pediatric sarcomas to define the utility of this marker in the routine diagnostic setting.

\section{MATERIALS AND METHODS}

Cases were retrieved from the files of the Laboratory of Pathology, National Cancer Institute, National 
Institutes of Health, Bethesda, Maryland and The Johns Hopkins Hospital, Baltimore, Maryland. A total of 119 tumors were evaluated, as summarized in Table 1. These included 69 cases of RMS (48 alveolar subtype/ARMS; 20 embryonal subtype/ERMS; one spindle cell RMS) and 50 other tumors, consisting of 16 ESFT, two ectomesenchymomas, six nephroblastomas, seven precursor hematopoietic neoplasms (including four lymphoblastic lymphomas), six desmoplastic small round cell tumors (DSRCT), five olfactory neuroblastomas (ONB), three neuroblastomas/ganglioneuroblastomas (NB/GNB), and five rhabdoid tumors. Formalin-fixed paraffin-embedded (FFPE) blocks or unstained sections were available for all of the cases. Routine morphologic and immunophenotypic studies were performed on $4-\mu \mathrm{m}$ thick hematoxylin- and eosin-stained sections. Staining for myogenin was also evaluated in a variety of fetal and adult tissues (including skeletal muscle, heart, liver, lung, kidney, gastrointestinal tract, spleen, lymph nodes, and brain).

Immunoperoxidase staining for myogenin was performed using the myf-4 antibody (L026, 1:10, mouse monoclonal, Novocastra Labs, Burlingame, $\mathrm{CA}$ ), according to the manufacturer's recommended protocol. Antigen retrieval was done in a pressure cooker for $40 \mathrm{~min}$ in $10 \mathrm{~mm}$ citrate buffer with $0.1 \%$ Tween 20 at $\mathrm{pH}$ 6.0. Slides were incubated overnight with the primary antibody, followed by detection using standard protocol on an automated immunostainer (Ventana Medical Systems, Tuscon, AZ), according to the manufacturer's instructions. Other immunoperoxidase stains included actin (HHF35, ENZO, Farmingdale, NY), desmin (D33), Der 11, NSE, 12E7 (p30/32mic2) (all from DAKO, Carpinteria, CA), AE1/3 (Boehringer Mannheim, Indianapolis, IN), and S100 (Biogenex, San Ramon, CA).

Staining for myogenin was graded from $1+$ to $4+$ as follows, based on the percentage of tumor cell nuclei staining positive: rare positive to $25 \%, 1+; 25$ to $50 \%, 2+; 50$ to $75 \%, 3+$; and 75 to $100 \%, 4+$.

\section{RESULTS}

Diagnosis was based on a combination of routine histologic and immunophenotypic criteria as well as, in some cases, correlation with ultrastructural and molecular findings. The alveolar RMS were composed of nests of undifferentiated primitiveappearing small round blue cells in solid nests or with a focal alveolar pattern. In some cases, cells with more abundant eosinophilic cytoplasm or multinucleated giant cell forms were evident. The PAX3/FKHR fusion transcript was detected in 16 of 22 cases evaluated by RT-PCR. The embryonal RMS were composed of round to spindled cells, often with eosinophilic cytoplasm, arranged in compactly cellular to more loose areas with a myxoid stroma.

The results of staining for myogenin are summarized in Table 1. Nuclear staining for myogenin was seen in all 48 ARMS (Fig. 1, 2, 3), 20 ERMS (Fig. 4), as well as in the spindle cell RMS. The results of grading in the RMS cases are summarized in Table 2. Grade $4+$ staining with 75 to $100 \%$ of nuclei staining positive was present in 44 ARMS, two cases showed $3+$ staining and in another two cases only $20 \%$ of nuclei stained, consistent with $1+$ staining. In contrast, in the ERMS group, 17 of 20 cases showed $1+$ staining, with the number of positive staining nuclei ranging from rare positive to $25 \%$. In three ERMS, staining was graded as $2+$, with 25 to $50 \%$ nuclei positive. None of the ERMS showed Grade $3+$ or $4+$ staining. The single case classified as spindle cell RMS showed $3+$ staining. In 66 of the 69 RMS, desmin-stained slides were available for review and all cases stained desmin-positive. In some of the more primitive appearing ARMS, however, the number of myogenin-positive cells clearly exceeded the desmin positivity (Fig. 5).

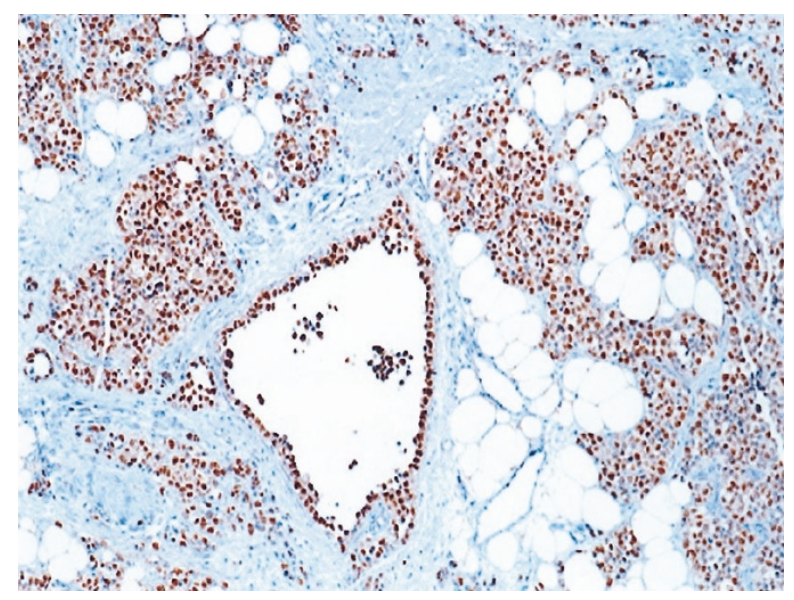

FIGURE 1. Tumor cells with intense nuclear positivity infiltrate in solid nests as well as line spaces in an alveolar rhabdomyosarcoma.

\section{Various Pediatric Tumors}

\begin{tabular}{lc}
\hline \multicolumn{1}{c}{ Diagnosis (\# of Cases) } & myf-4 \\
\hline Rhabdomyosarcoma (69) & $69 / 69+$ \\
Alveolar (48) & $48 / 48+$ \\
Embryonal (20) & $20 / 20+$ \\
Spindle cell (1) & $1 / 1+$ \\
Other pediatric sarcomas (50) & $0 / 50+$ \\
ESFT (16) & $0 / 14+$ \\
Nephroblastoma (6) & $2 / 6+^{a}$ \\
Ectomesenchymoma (2) & $2 / 2+$ \\
DSRCT (6) & $0 / 6+$ \\
Rhabdoid (5) & $0 / 5+$ \\
Precursor hematopoeitic neoplasms (7) & $0 / 7+$ \\
NB/GNB (4) & $0 / 4+$ \\
Olfactory neuroblastoma (5) & $0 / 5+$ \\
\hline
\end{tabular}

ESFT, Ewing's sarcoma family of tumors; DSRCT, desmoplastic small round cell tumor; NB/GNB, neuroblastoma/ganglioneuroblastoma.

$a$ These two nephroblastomas had myogenous differentiation. 


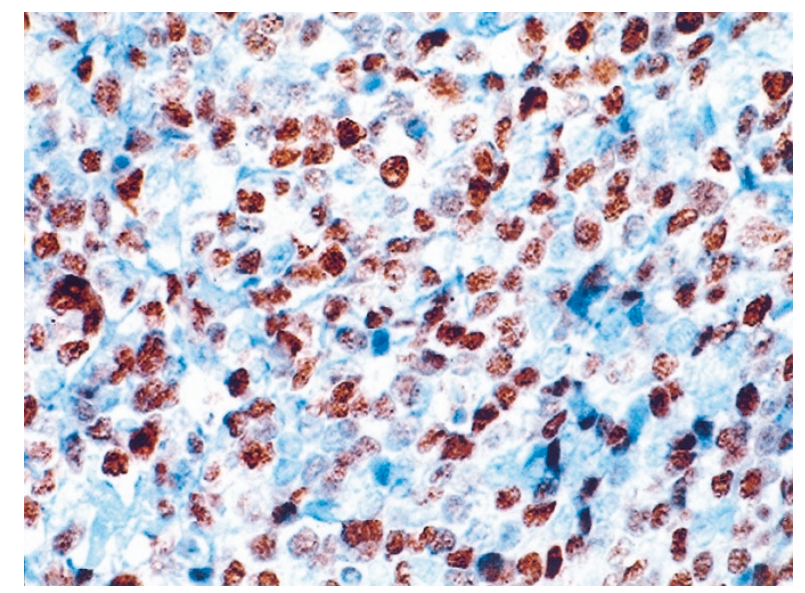

FIGURE 2. A case of solid alveolar rhabdomyosarcoma with the majority of cells showing nuclear staining (4+ staining).

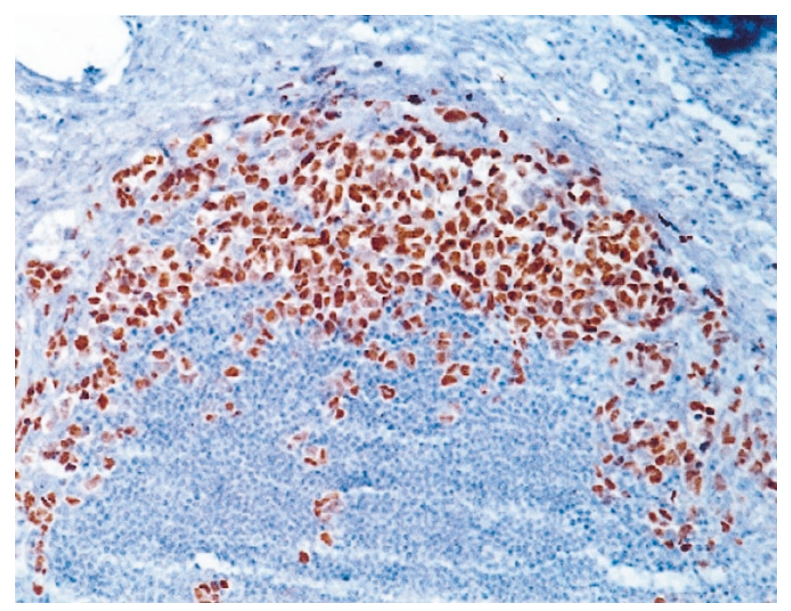

FIGURE 3. Staining for myf-4 in an alveolar rhabdomyosarcoma metastatic to the lymph node. Distinct nuclear positivity is seen in tumor cells within the subcapsular sinus whereas adjacent small lymphocytes are negative.

Myogenin-positive cells were identified in the two ectomesenchymomas, correlating with the desmin positive areas. In one case, the majority of the tumor was composed of undifferentiated cells that expressed only mic-2, whereas, in focal areas, the mic-2 positive cells also co-expressed myogenin, desmin and actin, as illustrated in Figure 6, A-C. Staining for myogenin (as well as desmin) was also noted in two of six nephroblastomas, and correlated with areas of myogenous differentiation (not shown). Staining for myogenin was absent in all other tumors studied (Table 1). Among the normal tissues evaluated for myogenin, positive staining was seen only in fetal striated muscle, as expected. All other tissues (adult and fetal) were negative.

\section{DISCUSSION}

The process of myogenesis involves the commitment and differentiation of pluripotent primitive

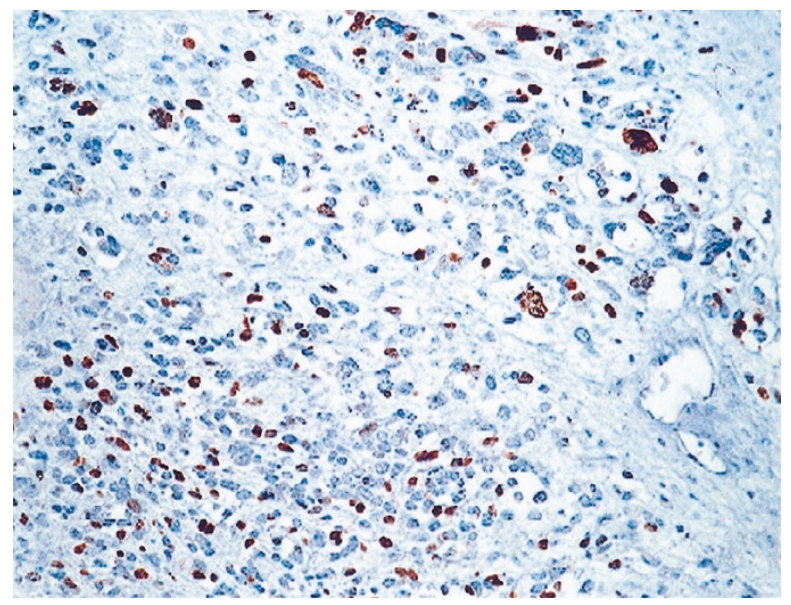

FIGURE 4. Scattered but distinctly myogenin-positive cells in an embryonal rhabdomyosarcoma. Nuclei in some of the multinucleated rhabdomyoblasts are also positive.

TABLE 2. Grading of Myogenin Expression in ARMS \& ERMS

\begin{tabular}{lcccc}
\hline \multirow{2}{*}{ Diagnosis } & \multicolumn{4}{c}{ Staining for myf-4 } \\
\cline { 2 - 5 } & Grade 1 & Grade 2 & Grade 3 & Grade 4 \\
\hline ARMS (48) & 2 & 0 & 2 & 44 \\
ERMS (20) & 17 & 3 & 0 & 0 \\
\hline
\end{tabular}
RMS.

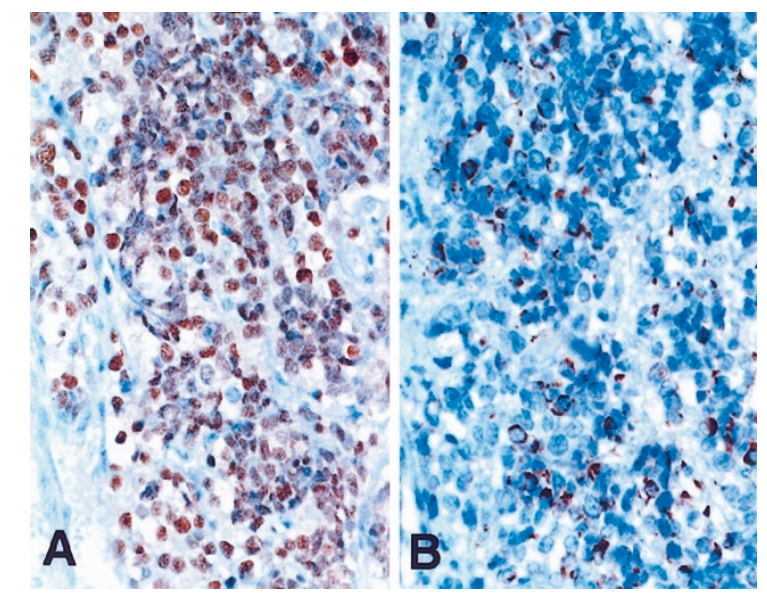

FIGURE 5. The number of tumor cells staining for myogenin (A) are more numerous than those marking with desmin (B) in a solid alveolar rhabdomyosarcoma.

mesodermal cells into skeletal muscle and is regulated by members of the MyoD family of genes. This family includes the MyoD1/myf-3, myogenin/myf4, $m y f-5$, and $M R F-4$ genes that encode for DNA binding proteins that act as transcription factors and bind to enhancer regions on muscle specific genes to induce their expression (10-16). Because the expression of these genes has been shown to be restricted to skeletal muscle, antibodies to MyoD1 and myogenin have been used to define lineage and confirm diagnosis of RMS in otherwise poorly dif- 


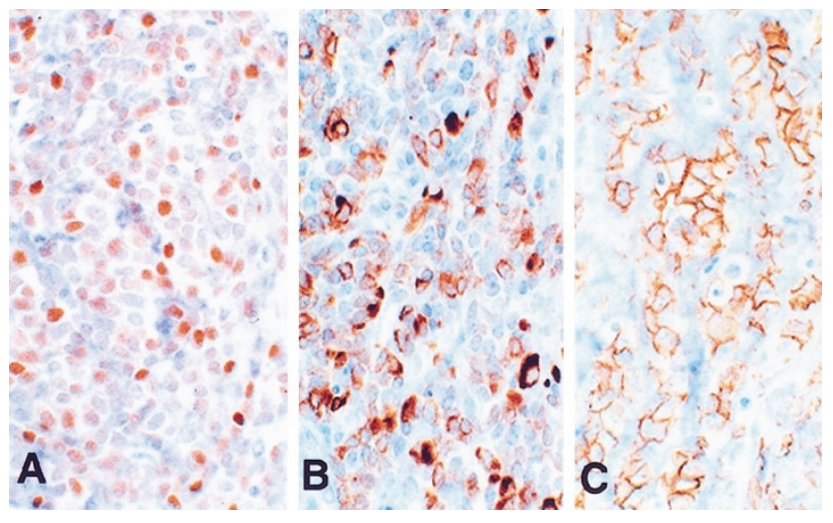

FIGURE 6. Composite figure of serial sections of an ectomesenchymoma showing co-expression of myogenin/myf-4 (A), desmin (B), and mic-2 (C) by the malignant cells. In most other areas (not shown in this figure), the tumor cells stained only for mic-2.

ferentiated sarcomas. Earlier studies required frozen tissue sections for evaluation of expression ( 1 , $2,4,5)$, but staining is now also possible on fixed tissue sections, and antibodies have recently become commercially available, allowing for wider use in the routine diagnostic setting $(6,8)$ Staining of fixed tissue sections with antibodies to MyoD1, however, often shows non-specific cytoplasmic staining, making interpretation of results difficult. This problem has been commented upon by several investigators $(6,7,8)$ and has also been our observation, particularly with the commercially available antibody, and tends to limit the clinical utility of this marker. This does not appear to be a problem with staining for myogenin, but, overall, relatively few cases of RMS have been analyzed for the expression of this protein. Moreover, the correlation of myogenin expression with different histologic subtypes of RMS has recently been suggested (9) but has not been further evaluated.

Our study included 69 cases of RMS and staining for myogenin was observed in all of these tumors. In prior studies, myogenin positivity has ranged from 71 to $91 \%$ of RMS, which is somewhat lower than what we observed. In the studies by Cui et al. (8) and Wang et al. (3), 16/20 (80\%) and 31/33 (91\%) RMS, respectively, were myogenin positive. The possibility that this slight difference could be attributable to the antibody used cannot be completely excluded because these two studies used an antibody different from ours: the clone 5FD antibody. However, in a third study, which included only seven RMS and was reported only in abstract form, Berezowski et al. (6) used the same antibody as ours but found only five of seven (71\%) cases to stain.

It is also possible that this apparent difference may relate to the subtypes of RMS evaluated in these prior studies and ours because a recent study has suggested that myogenin expression may correlate specifically with the alveolar subtype (9). Be- cause ARMS is less common than the embryonal type, the majority of tumors evaluated in prior studies have been of embryonal type. In fact, only $4 / 33$ and 6/20 RMS studied by Wang et al. (3) and Cui et al. (8), respectively, were of the alveolar subtype, whereas Berezowski et al. (6) did not specify subtype in their seven cases. Our study, in contrast, included a very large number of ARMS (48 cases) because our primary aim was to evaluate myogenin in the differential diagnosis of primitive round cell tumors of childhood, and it is the alveolar subtype that is often a differential diagnostic problem. In addition, given this recent observation by Palmer et al. (9) regarding strong myogenin expression specifically in ARMS, we wished to address this particular issue in a large number of cases. In their study, which was reported in an abstract, Palmer and colleagues evaluated 11 ARMS and 19 ERMS using a recombinant fusion protein from rat myogenin on cryostat sections. They found that all 11 ARMS showed strong myogenin expression, whereas the ERMS stained only weakly positive or were negative. Only 3/19 ERMS showed staining of a degree comparable to the ARMS cases (9). Our study essentially confirmed their observations in that we found consistent and strong expression of myogenin in virtually all our ARMS cases, with $46 / 48$ cases showing $3+$ to $4+$ staining and the two remaining cases showing $1+$ staining. Among our ERMS cases, only $3 / 20$ showed $2+$ staining, similar to the observations of Palmer et al. However, it should be emphasized that the 17 remaining ERMS in our study also showed distinct myogenin positivity, with intense individual nuclear staining, although the number of positive nuclei was clearly lower and ranged from a few to $25 \%$. This latter observation differs somewhat from that of Palmer et al. (9), who stated that their remaining ERMS cases were only weakly myogenin-positive or were negative, but did not elaborate further or specify the number of ERMS that were completely negative. Therefore, it is quite possible that the lower rate of myogeninpositive RMS reported in the earlier studies $(3,8)$ might be accounted for by the predominance of ERMS in these studies. Although this may suggest that staining for myogenin may not be as useful a marker for the ERMS group, our observations indicate that this is not necessarily true. Our study included cases where only one small tissue fragment among multiple contained tumor (ERMS), and distinctly positive cells, although few in number, could readily be identified in such fragments. Moreover, the myogenin positivity in these cases paralleled or exceeded the desmin positivity and was therefore quite helpful in confirming diagnosis.

It has been proposed that this strong correlation of myogenin expression with alveolar histology may relate to the stage of differentiation of the tumor 
cells. During the process of myogenesis, MyoD1 expression correlates with the initial stage of commitment to myogenesis: it is present in more undifferentiated cells, but becomes down regulated as differentiation proceeds. Myogenin expression, on the other hand, correlates with the stage of onset of differentiation as well as with maintenance of the differentiated state, and is therefore present in more differentiated cells $(14,15)$. Studies have compared muscle-specific gene expression profiles in RMS to the stages of normal fetal skeletal muscle development to investigate if phenotypic variations between RMS (embryonal versus alveolar) reflect malignant transformation of cells at specific stages of the myogenic pathway (11). Detection of myogenin transcripts in all ARMS (eight of eight) and the majority but not all ERMS (five of eight) in this study indicate that RMS resemble a relatively restricted segment of skeletal muscle development, limited at one end by the stage of commitment to myogenesis and, at the other, by the earliest overt stages of myogenesis. Within this scheme, the alveolar subtype phenotypically represents a restricted group, whereas the embryonal subtype encompasses a broader range of phenotypes and may show considerable overlap with the alveolar subtype. The two subtypes, however, may not necessarily represent malignant transformation of normal cells at different positions of myogenesis (11). The grade/pattern of expression of myogenin, as detected by routine immunohistochemical staining in our cases of ERMS and ARMS, is in keeping with this hypothesis. Palmer et al. (9) have further proposed that the strong expression of myogenin, which appears to be an integral part of alveolar histology, may be related to upstream activation of the myogenesis pathway by the PAX3/FKHR fusion protein unique to this tumor; however, this awaits further elucidation.

Interestingly, we observed distinct myogenin positivity in the two ectomesenchymomas included in our study, consistent with true rhabdomyoblastic differentiation. The distribution of myogeninpositive cells correlated with areas of desmin posi- tivity (as seen in Fig. 6), although, in one case, myogenin-expressing cells were more numerous than those positive for desmin. Although Northern blot analysis has demonstrated myogenin transcripts in three of five cell lines derived from these biphenotypic tumors in a prior study (17), other studies evaluating myogenin expression have not included examples of this rare tumor. Because these tumors can be composed almost entirely of undifferentiated primitive appearing cells that may stain for mic-2 (as in the case illustrated in Fig. 6), myogenin expression can be very helpful in distinguishing these from ESFT. Additionally, two Wilms' tumors with focal myogenous differentiation marked positively with both myogenin and desmin, confirming rhabdomyoblastic differentiation in these tumors, similar to a case previously reported by Wang et al. (3).

Desmin remains a very reliable and specific marker for RMS in the vast majority of cases (18), and we observed this in our study as well. However, we found that in several cases, particularly in the more primitive-appearing tumors, the myogeninpositive cells exceeded those staining for desmin, suggesting this marker may be especially useful in such tumors. In addition, rare ESFTs may mark with desmin. Such cases have been previously described in the literature $(3,19)$ and one of the 16 ESFTs in our study also stained positive for desmin. However, myogenin was clearly negative in this case, and served to exclude a diagnosis of RMS. Conversely, positivity for the mic-2 antigen may occasionally be present in RMS, and five ARMS in our series stained mic-2 positive. The inclusion of myogenin in an immunohistochemical panel for evaluating primitive round cell tumors such as these can thus be very useful in confirming rhabdomyoblastic lineage. Additionally, we found that other tumors, which can express desmin (such as DSRCT and renal/extra-renal rhabdoid tumors), were clearly negative for myogenin, suggesting that desmin expression is not indicative of rhabdomyoblastic differentiation in such cases - an observation made by others as well $(3,20)$. All other round

TABLE 3. Myogenin Expression in Various Tumor Types

\begin{tabular}{|c|c|c|c|c|c|c|c|c|c|c|c|c|c|c|}
\hline \multirow{2}{*}{ Reference } & \multirow{2}{*}{ Antibody } & \multirow{2}{*}{ Fixation } & \multicolumn{12}{|c|}{ Tumor Type, with \# of Cases Positive for Myogenin } \\
\hline & & & RMS & ESFT & NB & LMS & MFH & Wilms & DSRCT & ASPS & $\mathrm{Ca}$ & unspec & Rhabdoid & Ecto \\
\hline Wang et al. (3) & F5D & FFPE & $30 / 33$ & $0 / 26$ & $0 / 12$ & ND & ND & $1 / 1^{a}$ & ND & ND & ND & ND & ND & ND \\
\hline Wang et al. (7) & F5D & FFPE & ND & ND & ND & ND & ND & ND & ND & $0 / 12$ & ND & ND & ND & ND \\
\hline Berezowski et al. (6) & L026 & FFPE & $5 / 7$ & ND & ND & $0 / 10$ & $0 / 6$ & $1 / 7$ & ND & ND & $0 / 23$ & $0 / 29$ & ND & ND \\
\hline Gomez et al. (21) & F5D & FFPE & ND & ND & ND & ND & ND & ND & ND & $0 / 19$ & ND & ND & ND & ND \\
\hline Cui et al. (8) & F5D & FFPE & $16 / 20$ & $0 / 14$ & $0 / 2$ & $0 / 8$ & ND & ND & $0 / 1$ & ND & $0 / 2$ & ND & ND & ND \\
\hline Present study & L026 & FFPE & $69 / 69$ & $0 / 16$ & $0 / 3$ & ND & ND & $2 / 6^{b}$ & $0 / 6$ & ND & ND & ND & $0 / 5$ & $2 / 2$ \\
\hline
\end{tabular}

FFPE, formalin-fixed paraffin-embedded; ND, not done; RMS, rhabdomyosarcoma; ESFT, Ewing's sarcoma family of tumors; NB, neuroblastoma; LMS, leiomyosarcoma; DSRCT, desmoplastic small round cell tumor; ASPS, alveolar soft part sarcoma; MFH, malignant fibrous histiocytoma; Ca, carcinoma; Rhabdoid, renal/extrarenal rhabdoid; Ecto, ectomesenchymoma.

$a$ This was a Wilms' tumor with myogenous differentiation.

${ }^{b}$ These two tumors had myogenous differentiation, and were positive for myogenin as well as desmin. 
cell tumors evaluated in this study were clearly negative for myogenin as expected.

Although our study did not include spindle cell sarcomas or tumors such as alveolar soft part sarcoma, recent studies have reported these tumors to be consistently negative for myogenin $(6,7,21)$. The results of these studies are summarized in Table 3, and, in conjunction with our large series, serve to confirm the specificity of myogenin for rhabdomyoblastic differentiation in the routine diagnostic setting. False positive results do not occur.

In conclusion, we find staining for myogenin to be extremely sensitive and specific in defining rhabdomyoblastic lineage and a useful addition to the diagnostic immunohistochemical panel for small round blue-cell tumors. Although the literature suggests that myogenin expression parallels that of MyoD1, we believe that staining for the former is definitely superior in terms of quality and ease of interpretation. Furthermore, fixation does not appear to be a problem. The majority of our cases were derived from consultation material, with an inevitable variability in tissue fixation and processing; however, good results were obtained in all cases. Both ARMS and ERMS stain myogeninpositive, although, clearly, the number of cells staining in the latter subtype is fewer. This could be a potential pitfall when only small biopsies or needle core specimens are being evaluated.

\section{REFERENCES}

1. Dias P, Parham DM, Shapiro DN, Webber BL, Houghton PJ. Myogenic regulatory protein (MyoD1) expression in childhood solid tumors: diagnostic utility in rhabdomyosarcoma. Am J Pathol 1990;137:1283-91.

2. Dias P, Parham DM, Shapiro DN, Tapscott SJ, Houghton PJ. Monoclonal antibodies to the myogenic regulatory protein MyoD1: epitope mapping and diagnostic utility. Cancer Res 1992;52:6431-9.

3. Wang NP, Marx J, McNutt MA, Rutledge JC, Gown AM. Expression of myogenic regulatory proteins (myogenin and MyoD1) in small blue round cell tumors of childhood. Am J Pathol 1995;147:1799-810.

4. Tallini G, Parham DM, Dias P, Cordon-Cardo C, Houghton PJ, Rosai J. Myogenic regulatory protein expression in adult soft tissue sarcomas. A sensitive and specific marker of skeletal muscle differentiation. Am J Pathol 1994;144:693-701.

5. Wesche WA, Fletcher CDM, Dias P, Houghton PJ, Parham DM. Immunohistochemistry of MyoD1 in adult pleomorphic soft tissue sarcomas. Am J Surg Pathol 1995;19:261-9.

6. Berezowski K, Workman R, Kornstein MJ. Antibodies to myogenic regulatory proteins in the diagnosis of rhabdomyosarcoma [abstract]. Mod Pathol 1998;11:7A.

7. Wang NP, Bacchi CE, Jiang JJ, McNutt MA, Gown AM. Does alveolar soft part sarcoma exhibit skeletal muscle differentiation? An immunocytochemical and biochemical study of myogenic regulatory protein expression. Mod Pathol 1996;9: 496-506.

8. Cui S, Hano H, Harada T, Takai S, Masui F, Ushigome S. Evaluation of new monoclonal anti-MyoD1 and antimyogenin antibodies for the diagnosis of rhabdomyosarcoma. Pathol Int 1999;49:62-8.

9. Palmer H, Parham D, Chen B, Houghton P, Dias P. Strong immunohistochemical expression of myogenin correlates with alveolar histology in rhabdomyosarcoma [abstract]. Mod Pathol 1998;11:14A.

10. Arnold HH, Braun T, Bober E, Buchberger A, Winter B, Salminen A. Regulation of myogenin expression in normal and transformed myogenic cell lines. Symp Soc Exp Biol; 1992:37-51.

11. Tonin PN, Scrable H, Shimada H, Cavenee WK. Musclespecific gene expression in rhabdomyosarcomas and stages of human fetal skeletal muscle development. Cancer Res 1991;51:5100-6.

12. Clark J, Rocques PJ, Braun T, Bober E, Arnold HH, Fisher C, et al. Expression of members of the myf gene family in human rhabdomyosarcomas. Br J Cancer 1991;64:1039-42.

13. Parham DM. The molecular biology of childhood rhabdomyosarcoma. Semin Diagn Pathol 1994;11:39-46.

14. Jin P, Farmer K, Ringertz NR, Sejersen T. Proliferation and differentiation of human fetal myoblasts is regulated by PDGF-BB. Differentiation 1993;54:47-54.

15. Dias P, Dilling M, Houghton P. The molecular basis of skeletal muscle differentiation. Semin Diagn Pathol 1994;11:314.

16. Bouche M, Senni MI, Grossi AM, Zappelli F, Polimeni M, Arnold HH, et al. TPA-induced differentiation of human rhabdomyosarcoma cells: expression of the myogenic regulatory factors. Exp Cell Res 1993;208:209-17.

17. Sorensen PHB, Shimada H, Liu XF, Jerian FL, Thomas G, Triche TJ. Biphenotypic sarcomas with myogenic and neural differentiation express the Ewing's sarcoma EWS/FLI1 fusion gene. Cancer Res 1995;55:1385-92.

18. Dias P, Kumar P, Marsden HB, Morris-Jones PH, Birch J, Swindell R, et al. Evaluation of desmin as a diagnostic and prognostic marker of childhood rhabdomyosarcomas and embryonal sarcomas. Br J Cancer 1987;56:361-5.

19. Parham DM, Dias P, Kelly DR, Rutledge JC, Houghton P. Desmin positivity in primitive neuroectodermal tumors of childhood. Am J Surg Pathol 1992;16:483-92.

20. Ordonez NG. Desmoplastic small round cell tumor. II: an ultrastructural and immunohistochemical study with emphasis on new immunohistochemical markers. Am J Surg Pathol 1998;22:1314-27.

21. Gomez JA, Amin MB, Linden MD, Lee MW, Ro JY, Zarbo RJ. Is immunohistochemical (IHC) profile of myogenic regulatory proteins by myogenin and MyoD1 supportive of skeletal muscle lineage in alveolar soft part sarcoma (ASPS) [abstract]. Mod Pathol 1998;11:9A. 